\title{
Free Radicals
}

$\mathrm{T}$ HE General Discussion on "Free Radicals" held by the Faraday Society at Cambridge on September 28-30 was noteworthy for the large attendance of distinguished foreign visitors as well as for the number and range of the papers presented. It is impossible to do justice to all the contributions in a short review; what follows must be regarded as a personal impression of the more salient features of the discussion.

The definition of a free radical presents some difficulty. Probably the best 'working specification' is that of Wieland, quoted by Prof. T. M. Lowry in his opening paper. "Free radicals are complexes of abnormal valency which possess additive properties, but do not carry an electric charge and are not ions." This definition, like most of the others which have been attempted, is open to criticism; in particular, charged free radicals, for example, $\mathrm{CH}_{2}^{+}$, which contain an odd number of electrons, are familiar in positive ray work and were discussed later in the meeting.

In the opening paper, Prof. Lowry laid stress on the significance of free radicals for theories of valency and of the mechanism of organic reactions. In terms of the electronic theory, the splitting of a covalent link may be thought of as taking place unsymmetrically, giving two ions of opposite sign, or the link may be broken symmetrically so that each fragment of the molecule retains one of the electrons of the link and is a free radical. In the early days of the electronic theory of valency, it was thought that stable molecules might contain covalent linkages with any degree of polarity from a neutral bond like that between the carbon atoms in ethane to the polar bond in $\mathrm{NaCl}$. There is now a considerable body of physical evidence which indicates that the two types of linkage are fairly sharply marked off from one another. It is true that dipole moment determinations show that a single covalency can exhibit some degree of polarity, but this is always far short of the moment to be expected for the transference of an electron from one atom to another. The accumulating data on crystal structure and Raman spectra also give an experimental distinction between ionic and covalent linkages.

It follows that two main types of mechanism must be considered for organic reactions. Lapworth has shown that the formation of cyanhydrins is essentially an ionic reaction, and Lowry formulates it thus

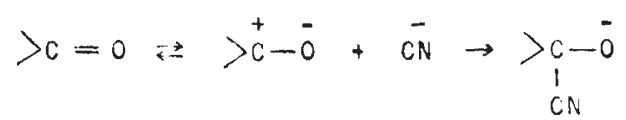

with an intermediate polarisation of the double bond. On the other hand, the thermal decom- position of hydrocarbons and many photochemical changes cannot be formulated as ionic reactions. Thus Norrish has found that the photochemical decomposition of methyl ethyl ketone follows the course :

$$
\left.\rightarrow \underset{\mathrm{C}_{2} \mathrm{H}_{5}}{\mathrm{C}=0} \rightarrow \underset{\mathrm{C}_{2} \mathrm{H}_{5}}{\mathrm{CH}_{3}}+\mathrm{CO} \rightarrow \begin{array}{l}
\mathrm{CH}_{2} \mathrm{C}_{6} \\
\mathrm{C}_{4} \mathrm{H}_{8} \\
\mathrm{C}_{10}
\end{array}\right\}+c 0
$$

The ultimate products are carbon monoxide and an equivalent mixture of nearly equal parts of ethane, propane and butane. It is evident that these products are most readily accounted for by the intermediate formation of the free radicals methyl and ethyl.

It is convenient here to mention a remarkable result obtained by Norrish for higher ketones. With methyl butyl ketone the products of photochemical decomposition are acetone and propylene.

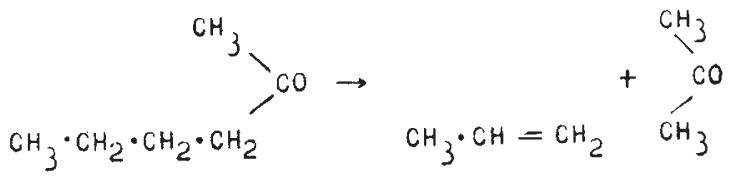

Since the radiation used is that absorbed by the carbonyl group, this reaction presents an interesting problem in molecular mechanics. It can be shown that nearly all the energy absorbed by the carbonyl group reappears as the energy of disruption of a bond in another part of the molecule.

Most of the free radicals met with in organic chemistry have one unsatisfied valency and therefore contain an odd number of electrons. These odd molecules should exhibit the paramagnetism due to an unbalanced electronic spin; this has been verified experimentally for inorganic free radicals, such as $\mathrm{NO}_{2}, \mathrm{ClO}_{2}$, etc., and more recently by Sugden for the typical organic free radicals, the ketyls $\left(\mathrm{R}_{2} \mathrm{C}-\mathrm{O}-\mathrm{K}\right)$. These substances have a high degree of stability and can be kept for an indefinite time; on the other hand, Paneth estimates the average life of a methyl radical as a few thousandths of a second. Free radicals thus fall into two groups, the stable type with a long life, and the evanescent type with a very short average life.

Many organic radicals of the first type have been prepared since Gomberg, in 1900, discovered triphenylmethyl. A valuable review of this series of compounds was given by Prof. Ziegler. The factors which favour the dissociation of the hexaaryl ethane molecule appear to be the unsaturation and the volume of the substituents, and of these, volume appears to be more important. Thus pentaphenyl ethyl and pentaphenyl cyclopentadienyl appear to exist entirely as the free radical. For the latter substance an interesting symmetrical electronic formula (I) has been suggested by Löwenbein in which a ring is formed by five 
three electron links. The introduction of substituents containing triple bonds appears to have little effect in promoting the dissociation of the ethane linkage.

$$
\begin{aligned}
& \ddots_{c}^{\stackrel{P h}{c}} \cdot{ }_{c: P h}
\end{aligned}
$$

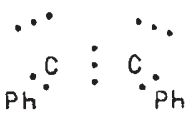

$$
\begin{aligned}
& \text { I }
\end{aligned}
$$

The number of radicals of the triphenyl methyl type now known is very large and progress is to be expected rather in the accurate physico-chemical study of known complexes than in the preparation of new substances. Ziegler and his co-workers have already developed accurate methods for determining the energy changes which accompany the dissociation of hexa-aryl ethanes; these have so far only been applied to hexaphenyl ethane. The rate of dissociation has been followed by three reactions (with $\mathrm{I}_{2}, \mathrm{NO}$, and $\mathrm{O}_{2}$ ) and is found to be constant in a number of different solvents. The temperature coefficient of reaction velocity gives a heat of activation of 19 Cal. This is much less than the heat of splitting of the usual C-C bond, namely, c. 70 Cal. Ziegler has also worked out an accurate optical method of determining the degree of dissociation of hexaphenyl ethane in various solvents. The temperature coefficient of the dissociation constant gives a heat of dissociation of about 11 Cal.

These measurements are of interest in connexion with a quantum mechanical theory of triphenyl methyls contributed by Prof. E. Huckel. In Huckel's theory of benzene the stability and planar configuration of the ring are ascribed to resonances between six electrons, one from each carbon atom, which are designated $p_{h}$ electrons. In hexaphenyl ethane the aromatic nuclei must, on stereochemical grounds, be arranged at or near tetrahedral angles around the central carbon atoms, and with this arrangement it is not possible to have interaction between the $p_{h}$ electrons of one aromatic nucleus and another. In triphenyl methyl, however, the three phenyl groups can approach a planar configuration with links to the central carbon atom at $120^{\circ}$. A marked interaction between the electrons of the three nuclei can then occur which stabilises the free radical. This energy of stabilisation must be subtracted from the heat of rupture of the $\mathrm{C}-\mathrm{C}$ bond to give the heat of activation of the hexaphenyl ethane molecule. Hückel terms the energy of stabilisation the "static bond strain", and computes its value approximately for typical substituted ethanes. With six aromatic substituents the static bond strain is 55 Cal., in fair agreement with Ziegler's experimental results. The strain decreases rapidly with the number of aromatic substituents ; for one phenyl group in the molecule it is only $12 \mathrm{Cal}$.
Another discussion of free radicals in terms of quantum mechanics was contributed by Prof. J. E. Lennard-Jones, who applied the method of molecular orbitals to investigate the structure of the $\mathrm{CH}_{3}, \mathrm{CH}_{2}$, and $\mathrm{CH}$ radicals. Particular interest attaches to the structure of $\mathrm{CH}_{2}$, which has a singlet normal state and not, as might have been expected, a triplet state. The production of $\mathrm{CH}_{2}$ from $\mathrm{CH}_{3}$ involves not only the removal of a hydrogen atom but also an "energy of reorganisation" of the electrons in the $\mathrm{CH}_{2}$ residue so that the net spin becomes zero. A rather cruder way of expressing this result (since it refers to a particular atom and not to the molecule as a whole) is to state that whilst $\mathrm{CH}_{3}$ contains quadrivalent carbon, $\mathrm{CH}_{2}$ contains bivalent carbon. This energy must play an important part in many chemical and photochemical changes. Thus carbon monoxide is known from spectroscopic evidence to be ${ }^{1} \Sigma$ with bivalent carbon, whilst the carbonyl group in ketones may be regarded as containing quadrivalent carbon. Hence the photochemical change studied by Norrish in which carbon monoxide is formed from the lower ketones must involve an energy of reorganisation.

A number of papers were contributed on the chemical reactions of radicals of short life. The simplest of all free radicals is, of course, atomic hydrogen, which can be produced in high concentrations by a discharge through hydrogen at pressures of less than $1 \mathrm{~mm}$. This radical has the comparatively long life of about one second, and some recent work on its chemical properties and those of atomic oxygen was described by Hartek. At low temperatures (liquid air or hydrogen) some very striking results have been obtained. With oxygen, atomic hydrogen gave high yields of hydrogen peroxide, with mercury a solid product containing 70 per cent of $\mathrm{HgH}$, and with ethylene ethane was formed. In the last reaction, the apparatus was capable of producing 500 c.c. of ethane, measured at atmospheric pressure, in 15 minutes.

The use of free methyl and ethyl to synthesise organometallic compounds was described by Paneth. Amongst the compounds isolated was the long sought for antimony analogue of cacodyl, $\left(\mathrm{CH}_{3}\right)_{2} \mathrm{Sb} . \mathrm{Sb}\left(\mathrm{CH}_{3}\right)_{2}$. This substance is spontaneously inflammable in air and melts at $17 \cdot 5^{\circ} \mathrm{C}$. In the liquid state and in solution it is nearly colourless, but on solidification becomes bright red. Horn, Polanyi and Style described experiments showing that methyl and ethyl are formed by the action of sodium on alkyl halides at $200^{\circ}-$ $300^{\circ} \mathrm{C}$. The radicals were detected in two ways : (a) by using them to initiate the chain reaction between hydrogen and chlorine; and $(b)$ by interaction with iodine to give alkyl iodides which were frozen out and determined by the usual analytical methods. The interesting radical methylene, $\mathrm{CH}_{2}$, was obtained by Belchez as the primary product of the thermal decomposition of methane on a platinum surface at $1,100^{\circ} \mathrm{C}$. and $0.1 \mathrm{~mm}$. pressure. The radical was identified by reaction 
with an iodine mirror and the subsequent formation of the additive compound $\mathrm{CH}_{2} \mathrm{I}_{2} \mathrm{Hg}_{2}$ of melting point $230^{\circ} \mathrm{C}$.

Finally, a number of papers were devoted to the study of free radicals in electric discharges by spectroscopic or positive ray methods. Of these there is only space to mention the important report by Mecke on "Free Radicals and Spectroscopy". Spectroscopic methods not only detect the presence of free radicals but also for diatomic radicals give exact information about their dimensions and the strength of the interatomic linkage. It is true that polyatomic radicals and molecules give spectra of such complexity that a full analysis is difficult, but Mecke pointed out that there is a good deal of evidence for regarding simple radicals as the bricks from which larger molecules are built up. Their dimensions show little change when they form part of a larger molecule, and similar linkages have similar binding energies in diatomic radicals and in larger collections of atoms. Thus the accurate spectroscopic data for diatomic radicals and molecules can be applied with little modification to the discussion of the dimensions and strength of linkages in more complex systems.

S. S.

\section{Obituary}

Prof. P. Ehrenfest

$\mathrm{T}$ HEORETICAL physics has lost one of its ablest and most enthusiastic exponents through the death on September 25 last of Paul Ehrenfest, professor of theoretical physics in the University of Leyden. Born in Vienna in 1880, he studied first under Boltzmann. From Vienna he went to Göttingen; there he made the acquaintance of his future wife (Mrs. T. Ehrenfest-Afanashewa). In collaboration with Mrs. Ehrenfest, he undertook a thorough critical investigation of the principles underlying the kinetic theory of matter, a problem which had fascinated him ever since his Vienna days. The elucidation of certain paradoxes in Boltzmann's $H$-theorem (1907) and the extensive article on statistical mechanics in the "Enzyklopadie der mathematischen Wissenschaften" (1912) testify to the fertility of their combined activity. The latter work was written in Russia, where the Ehrenfests lived for several years.

In 1912, Ehrenfest was called to Leyden to take the chair of theoretical physics on the retirement of H. A. Lorentz. With the exception of minor interruptions (lecturing trips to the United States and Russia) he worked and taught at Leyden until his death. Of his scientific work of this period we may mention his prediction of the interference patterns produced by $\mathrm{X}$-rays travelling through a diatomic gas (1915), his work on the adiabatic hypothesis in the quantum theory $(1916$; his own delightful survey of this topic in Die Naturwissen. schaften, 1923, should be consulted) and his theorem on the propagation of wave packets in quantum mechanics (1927).

Ehrenfest's intellectual enthusiasm and his innate desire to communicate to others (especially the younger workers) everything he understood and also every question which he felt unable to answer, was a novel experience to the phlegmatic sons of Holland. In contrast to the usual educational principles, he infected his students with his own enthusiasm and rushed with them to the outposts of the empire of physics, where the fighting against the great unknown--relativity and quantum theory--was going on. But at the same time, he did not forget to take them to an occasional tower, from which he could show and explain to them in his masterly way the domains already conquered. Finally, he taught them the importance of careful attention to details; his own love for detail and his perseverance in following up minor points until every elementary logical thread was unravelled, set us an example of highest scientific, value.

Ehrenfest's success as a teacher was inseparably connected with his critical vein. How can a teacher expound what he does not himself fully understand? In harmony with his ideals, he maintained, throughout his life, that the obvious rules of scientific communication should be kept, namely, that a lecturer or author should understand what he says or writes and should be understandable by his audience. Readers of NATURE who have not had the privilege of meeting Ehrenfest may deem such an ideal quixotic; those who have will remember that, besides his honesty and his seriousness, he had a ready wit and boyish laughter at his disposal. Lectures where the audience was expected not to interrupt were, as a rule, a bore to him, especially if he was himself the lecturer.

Those who came in personal contact with Ehrenfest were struck by the richness of his personality, every domain of true spiritual culture being dear to him. The profoundly human and altruistic sentiment, which was always in the background of his thoughts and actions, made him a valued friend to many and also brought him many devoted friends who-in his Iater years-would have liked to see him happy more often.

H. A. Kramers.

WE regret to announce the following deaths:

Mr. David Miller Muir, a well-known radiologist of Exeter, who worked under Sir J. J. Thomson at the Cavendish Laboratory, Cambridge, before taking up the medical aspects of his subject, on October 18, aged forty-six years.

Dr. Christine Murrell, a member of the Council of the British Medical Association and first woman member of the General Medical Council, on October 18, aged fifty-nine years. 